\title{
Primeira ocorrência de Spalangia cameroni Perkins (Hymenoptera: Pteromalidae) como parasitóide de Ornidia obesa Fabricius (Diptera: Syrphidae) no Brasil
}

\author{
First occurrence Spalangia cameroni Perkins \\ (Hymenoptera: Pteromalidae) as a parasitoid of Ornidia obesa \\ Fabricius (Diptera: Syrphidae) in Brazil
}

\author{
Carlos Henrique Marchiori1*; Jarbas Magno Miranda²; \\ Layara Alexandre Bessa; André Luis Ribeiro
}

\begin{abstract}
Resumo
Este estudo relata a primeira ocorrência de Spalangia cameroni Perkins (Hymenoptera: Pteromalidae) como parasitóide de Ornidia obesa Fabricius (Diptera: Syrphidae) encontrado em granjas de aves poedeiras em Morrrinhos, Goiás, Brasil. Amostras de fezes de galinha foram coletadas em intervalos de duas semanas de sua exposição, levadas para o laboratório para extração das pupas pelo método de flotação. As pupas foram individualizadas em cápsulas de gelatina e mantidas até a emergência dos dípteros e/ou parasitóides. A porcentagem de parasitismo foi de 9,5\%.

Palavras-chave: Hymenoptera, fezes de galinha, inimigo natural, controle biológico, Goiás
\end{abstract}

\begin{abstract}
This study reports, for the first time, the occurrence of Spalangia cameroni Perkins (Hymenoptera: Pteromalidae) as parasitoid of Ornidia obesa Fabricius (Diptera: Syrphidae) in poultry farms in Morrinhos, Goiás, Brazil. Manure chicken samples, collected at two weeks intervals, were taken to the laboratory and the pupae were extracted by water flotation. Each pupa was placed in capsules of gelatin until the emergence of dipterous or their parasitoids. The parasitism percentage was $9.5 \%$.

Key words: Hymenoptera, chicken dung, natural enemy, biocontrol, Goiás
\end{abstract}

\footnotetext{
1 Biólogo, Doutor em Ciências Biológicas; Prof. do Instituto Federal de Educação, Ciência e Tecnologia Goiano - Campus Morrinhos, E-mail: chmarchiori@yahoo.com.br

2 Licenciado em Ciências Agrárias; Prof. do Instituto Federal de Educação, Ciência e Tecnologia Goiano - Campus Morrinhos.

* Autor para correspondência
} 
Para obter um controle satisfatório destes insetos, um programa de manejo deve integrar métodos culturais, químicos e biológicos. Os agentes de controle biológico terão maior chance de serem bem sucedidos se forem mais adaptados à região, à época do ano e ao tipo de esterco do local (COSTA; BERTI FILHO; SILVEIRA NETO, 2004).

A espécie Ornidia obesa Fabricius (Diptera: Syrphidae) é extensivamente distribuída nas Américas e disseminou-se amplamente no Velho Mundo no século XIX através do comércio (MORALES; KÖHLER, 2004). Os adultos de O. obesa abrigam bactérias de importância para a saúde pública (PODLIPAEV; NAUMOV, 2000; MORALES; KÖHLER, 2004).

Spalangia cameroni Perkins (Hymenoptera: Pteromalidae) é considerado um parasitóide solitário de pupários numerosos Diptera nas famílias Anthomyiidae, Muscidae, Otitidae e Sarcophagidae (BAI; SANKARAM, 1977; BLUME, 1984; SERENO; NEVES, 1993).

O objetivo deste trabalho é relatar a primeira ocorrência de $S$. cameroni parasitando $O$. obesa no Brasil.

O experimento foi realizado na granja do CEFET-UNED, Morrinhos em Goiás. As fezes coletadas originaram-se de 80 aves da linhagem "Hyline". A granja é composta por dois galpões de $15 \mathrm{~m}$ de comprimento por $5 \mathrm{~m}$ de largura e $5 \mathrm{~m}$ de altura, com uma porta de um metro de largura por dois metros de altura que permite a entrada e saída de funcionários. O galpão é formado por duas fileiras de gaiolas construídas de armação de metal contendo duas aves por gaiola. As fileiras com as gaiolas ficam separadas por um corredor de cimento de $2 \mathrm{~m}$ de largura e as mesmas ficam suspensas do chão a 50 centímetros de altura. O piso abaixo das gaiolas e ao redor é constituído por terra. As fezes acumuladas sob as gaiolas variavam de umidade, de consistência pastosa ou firme. Em um galpão as fezes frescas foram coletadas imediatamente após a emissão e colocadas em 4 bacias de 30 $\mathrm{cm}$ de diâmetro por 12 centímetros de altura, as quais foram deixadas no próprio ambiente, em local seco. As fezes permaneceram no galpão por 15 dias, posteriormente, as bacias foram retiradas e levadas para o laboratório para a extração das pupas pelo método da flotação. As pupas foram retiradas com o auxílio de uma peneira, contadas e individualizadas em cápsulas de gelatina (número 00) até a emergência dos dípteros e/ou dos seus parasitóides. Os parasitóides e os dípteros emergidos foram identificados com auxílio de um microscópio estereoscópio e, posteriormente, conservados em álcool 70\%.

A porcentagem de parasitismo foi calculada dividindo o número de pupas parasitadas pelo número total de pupas coletadas, multiplicando o resultado por cem.

No período de agosto a dezembro de 2007 coletaram-se 21 exemplares de O. obesa, dos quais emergiram dois exemplares do parasitóide $S$. cameroni. A porcentagem de parasitismo foi de $9,5 \%$.

No Brasil, $S$. cameroni foi encontrada parasitando Chrysomya putoria (Wiedemann) (Diptera: Calliphoridae), Fannia trimaculata (Stein) (Diptera: Fanniidae) Musca domestica L. (Diptera: Muscidae), Muscina stabulans (Fallèn) (Diptera: Muscidae), Stomoxys calcitrans (L.) em fezes de galinha (COSTA et al., 1992; SERENO; NEVES, 1993; MARCHIORI, et al., 2003).

Em Goiás, O. obesa foi encontrada parasitada com o parasitóide Pachycrepoideus vindemmiae (Rondani) (Hymenoptera) também em fezes de galinha. (MARCHIORI et al., 2007).

Microhimenópteros parasitóides da família Pteromalidae são naturalmente coletados emergindo de pupários de dípteros que proliferam no esterco de aves e algumas espécies podem ser utilizadas como controladores biológicos (SERENO; NEVES, 2003). 
Não há relatos de $S$. cameroni parasitando $O$. obesa no Brasil e no mundo. Este trabalho registra a primeira ocorrência do parasitóide $S$. cameroni parasitando O. obesa no Brasil.

\section{Referências}

BAI, M. G.; SANKARAN, T. Parasites, predators and other arthropods associated with musca domestica and other flies breeding in bovine manure. Entomophaga, Paris, v. 22, n. 2, p. 163-167, 1977.

BLUME, R. R. Parasites of Diptera associated whith bovine droppings on a pasture in east Central Texas. Southwest Entomologist, Garden City, v. 11, n. 3, p. 215222, 1984.

COSTA, V. A.; BERTI FILHO, E.; SILVEIRA NETO, S. Parasitóides (Hymenoptera: Chalcidoidea) de moscas sinantrópicas (Diptera: Muscidae) em aviários de Echaporã, SP. Arquivos do Instituto Biológico, São Paulo, v. 71, n. 2, p. 203-209, 2004.

COSTA, V. A.; GUIMARÃES, J. H.; TUCCI, E. C.; SANTOS, A. M. M. Parasitóides associados a dípteros sinantrópicos de Granjas de aves de postura no Estado de São Paulo, Brasil. Revista Brasileira de Parasitologia Veterinária, Seropédica, v. 1, n. 1, p. 55-59, 1992.
MARCHIORI, C. H.; RODRIGUES, B. G.; SOUZA, L. R.; SILVA, C. F.; VIEIRA, T. C.; TIRONE, H. C.; MARCUS, C. G. Pachycrepoideus vindemmiae (Rondani) (Hymenoptera: Pteromalidae) como parasitóide de Ornidia obesa Fabricius (Diptera: Syrphidae) no Brasil. Instituto Biológico, São Paulo, v. 74, n. 4, p. 361-362, 2007.

MARCHIORI, C. H.; SILVA FILHO, O. M.; BORGES, M. P.; MORAES, P. C.; ARANTES, S. B. Parasitóides de musca domestica L. (Diptera: Muscidae) de Itumbiara, Goiás, Brasil. Revista de Patologia Tropical, Goiânia, v. 32, n. 2, p. 263-266, 2003.

MORALES, M. N.; KÖHLER, A. Ornidia Lepeletier \& Serville, 1828 (Diptera, Syrphidae) no Estado do Rio Grande do Sul, Brasil: distribuição e preferência floral. Revista Brasileira de Zoociências, Juiz de Fora, v. 6, n. 1, p. 93-102, 2004.

PODLIPAEV, S. A.; NAUMO, V. A. D. Colonies of trypanosomatids on agar plates: the tool for differentiation of the species and isolates. Protistology, Saint Petersburg, v. 1, n. 3, p. 113-119, 2000.

SERENO, F. T. P. S.; NEVES, D. Microhimenópteros (Pteromalidae) parasitóides de diptera (Muscidae, Otitidae) em uma granja de bovinos em Igarapé, Estado de Minas Gerais, Brasil. Revista Brasileira de Entomologia, Curitiba, v. 37, n. 3, p. 563-567, 1993. 
